\title{
Optimal operation of water distribution networks by predictive control using MINLP
}

\author{
C Biscos ${ }^{1}$, M Mulholland ${ }^{2 *}$, M-V Le Lann ${ }^{3}$, CA Buckley ${ }^{1}$ and CJ Brouckaert ${ }^{1}$ \\ ${ }^{1}$ Pollution Research Group, School of Chemical Engineering, University of Natal, Durban, 4041, South Africa \\ ${ }^{2}$ School of Chemical Engineering, University of Natal, Durban, 4041, South Africa \\ ${ }^{3}$ Laboratoire d'Analyse et d'Architecture des Systèmes, 7 avenue du Colonel Roche, 31077 Toulouse Cedex 4, France \\ or Institut National des Sciences Appliquées, Dépt. G.E.I. , 135 av. de Rangueuil, 31077 Toulouse Cedex 4, France
}

\begin{abstract}
This paper presents an approach for the operational optimisation of potable water distribution networks. The maximisation of the use of low-cost power (e.g. overnight pumping) and the maintenance of a target chlorine concentration at final delivery points were defined as important optimisation objectives. The first objective is constrained by the maintenance of minimum emergency volumes in all reservoirs, while the second objective would include the minimisation of chlorine dosage and re-dosage requirements. The combination of dynamic elements (e.g. reservoirs) and discrete elements (pumps, valves, routing) makes this a challenging predictive control and constrained optimisation problem, which is being solved by MINLP (Mixed Integer Non-linear Programming). Initial experimental results show the performance of this algorithm and its ability to control the water distribution process.
\end{abstract}

Keywords: drinking water, mixed integer non-linear programming, model predictive control

\section{Introduction}

Durban is the busiest port in Africa, one of the largest economic bases in Southern Africa, and has strong recreational and tourism potential. It is the primary economic centre of the Kwazulu-Natal region. Because of the arrival of rural people from other parts of KwaZulu-Natal, the number of inhabitants in the municipality is increasing continuously creating additional stress on existing infrastructure. This region is also affected by the disastrous impact of the HIV/AIDS epidemic. This is likely to cause large changes in the current population growth rate in the short term, and consequently should lead to a reconsideration of future needs for services delivery. The optimal use of existing infrastructure, such as water distribution systems, seems to be the best solution for the present and that is where this project can play a part.

All of the water services functions of the Metropolitan Area have been included into a single entity named eThekwini Water Services. The network under their supervision extends over a region covering $85 \mathrm{~km}$ along the coast and $60 \mathrm{~km}$ inland. Approximately $800 \mathrm{~km}$ of bulk mains convey treated water to 220 storage reservoirs within the Metropolitan Area. Water is further distributed via $4500 \mathrm{~km}$ of pipes, which radiate outwards from the storage reservoirs. The reservoir volumes are measured on-line and sent to a central SCADA system where all of the relevant data are stored. To deliver potable water with an adequate pressure, even in remote parts of the system, there are 235 pump-stations. It is possible to actuate these remotely by telemetry from the central control room. Approximately 160 test points in the piped potable water distribution system are continuously monitored by eThekwini Water Services to ensure compliance with international health

\footnotetext{
* To whom all correspondence should be addressed.

푱 +27312603123; fax: +27312601118; e-mail: MULHOLLA@nu.ac.za Received 21 January 2003; accepted in revised form 22 May 2003.
}

norms promulgated by the World Health Organisation (WHO) in the third edition (2003) of the Guidelines for Drinking Water Quality (http://www.who.int/water_sanitation_health/dwq/guidelines2/en/).

Optimisation of water distribution systems has gained much attention in the past few decades. Research is spurred by the complexities associated with the operation of multiple interconnected reservoirs, which still exceeds the capabilities of existing optimisation tools in finding solutions easily (Teegavarapu and Simonovic, 2002). Mathematical programming techniques are one of many tools available to engineers for determining optimal configurations of a particular process. Recent developments in this field have given rise to new methods for determining the optimal operation of a process. Optimisation of reservoir operations has recently received much attention (Mousavi and Ramamurthy, 1999). Numerous algorithms are being tested on distribution systems by researchers to get the most reliable solutions, using the least computational time possible. Linear programming (LP), nonlinear programming (NLP), mixed-integer linear programming (MILP), mixed-integer nonlinear programming (MINLP), as well as fuzzy logic (FL), stochastic dynamic programming (SDP) and the simulated annealing (SA) are the most promising methods.

Until recently, the topic of optimisation in water systems in the literature has been mainly focused on the design of optimised configurations for pipe-interconnected reservoirs. For more information on this, the reader can refer to Schaake and Lai, 1969; Alperovits and Shamir, 1977; Quindry et al., 1981; Goulder and Morgan, 1985; Fujiwara et al., 1987; Woodburn et al., 1987; Lansey and Mays, 1989; Kessler and Shamir, 1989, 1991; Duan et al., 1990; Eiger et al., 1994; Kim and Mays, 1994; Cunha and Sousa, 1999.

Although most of the research in the field of water distribution optimisation has concerned the optimal design of new networks, a few researchers have tried to develop new techniques for the operational optimisation of existing infrastructures. The objective 
is mainly to generate control strategies ahead of present time, using predictive techniques, to guarantee a competent network service, while simultaneously achieving certain quality objectives. Minimisation of supply and pumping costs, maximisation of water quality and leak prevention are among the main targets (Cembrano et al., 2000). In most networks, this optimisation problem is equivalent to optimising the scheduling of pumps. Creasey (1988) reviewed the appropriate mathematical techniques to solve the problem of operational optimisation for water distribution networks, insisting particularly on the pump-scheduling problem. He concludes that better pump scheduling could save $£ 10$ million (1988) a year to the UK water industry alone. Although he recognises that there is a range of suitable scheduling tools, he also states that integer-based programming seems to be the only way to achieve these savings for a wide range of network sizes and complexities. This optimisation problem is usually large, and known to be non-linear. The non-linear nature originates from pump and pipeline pressure characteristics. Unfortunately, with the limited available expertise, which existed in integer programming at the time of the first studies on operational optimisation of water distribution systems, alternative methods had to be developed. It appears that most of these initial approaches were based on dynamic programming. Examples of application of dynamic programming on small-scale systems can be found in Rao and Bree (1977), Wood and Rayes (1981), Goldberg and Kuo (1987), and Coulbeck (1988).

The first successful application of dynamic programming on an existing large-scale system appeared in the mid-seventies, with Fallside and Perry (1975). These two researchers derived an hierarchical price-decomposition algorithm for the optimisation of a water supply system in the Cambridge area (United Kingdom). The objective of their optimisation was to provide an automatic scheduling of pumps, to meet consumption demand at minimum cost. Their method became operational at the end of the 1980s, after successive improvements (Coulbeck, 1977). At the end of the process of validation of the method, Fallside (1988) enumerated the requirements to use this methodology for systems that are more general than the initial application. In Spain, Solanas (1974) created an approximation of an existing dynamic programming algorithm, which was improved until 1988, and finally successfully implemented on the Barcelona distribution network (Solanas and Montoliu, 1988). In the 1980s, one of the most relevant applications was the implementation of the model developed by Carpentier (1983), for the southern areas of the Parisian distribution network. The use of Carpentier's method has since been successful in other areas of the Parisian distribution system. For instance, Alla and Jarrige (1988) present economic results concerning the use since 1985 of this system on the western areas of Paris. On the positive side, they highlight that the benefits, which can be realised with the optimised sequencing, amount to between 0 and $5 \%$ of the total operational cost of this particular part of the network. However, on the negative side, these savings are achieved at the expense in terms of safety, since reservoir levels often reach their lower emergency levels during periods when the electricity tariffs are high. Furthermore, pump and valve operations occur very often, which significantly increase operator workload, since operators were required to implement the changes.

At the end of the 1980 s, the need to develop optimisation approaches that are more general became important. LP appeared to be one of the promising methods to solve more general operational optimisation problems. With the availability of LP algorithms that were more robust and efficient, several papers on the subject were published. Jowitt et al. (1988), or Jowitt and
Germanopoulos (1992) produced the most significant applications in the field of LP techniques applied to the pump-scheduling problem. However, it appeared that the accuracy of LP-based models could be quite poor. Consequently, one of the latest approaches consists of taking into account the non-linear relationships, which are part of any pump-scheduling problem.

The appearance of improved non-linear programming (NLP) algorithms, convinced researchers to rather apply NLP techniques to solve the operational optimisation problem. Chase and Ormsbee (1989), Lansey and Zhong (1990) and Brion and Mays (1991) linked network-simulation models with non-linear optimisation algorithms to determine optimal operations. Yu et al. (1994) also proposed a method based on NLP to determine the optimal operation of general water distribution systems containing multiple sources and reservoirs. Since NLP techniques are recent, few references are available in the literature to prove successful application of these methods to existing installations. The work conducted in this field is still at the stage of research. For instance, a number of recent European research programmes have attempted to produce tools applicable to a variety of water utilities across Europe. The most advanced, WATERNET, aims to demonstrate the benefits of introducing optimisation techniques in the water distribution sector. Cembrano et al. (2000) are part of this research programme. Their work proves the advantages of optimal control methods on the water distribution network of a Portuguese district. However, although they successfully integrated their model within the existing supervisory control system, they were unable to obtain integer strategies for pumping operations, which is the final objective of the WATERNET research programme.

In this paper, a method for real-time optimal operation of a water distribution network is presented. The paper proposes a new approach to address this important issue. The maximisation of the use of low-cost power and the maintenance of the target chlorine concentration at final delivery points were defined as the main optimisation objectives of this problem. The first objective is constrained by the maintenance of the operational volumes of reservoir within two boundaries: the minimum emergency volume on the one hand, and the maximum capacity volume on the other hand. Minimising the chlorine dosage and re-dosage requirements would impact on the second objective.

The minimisation of the operational costs can be obtained by using optimal planning, based on predictive techniques that will allow the best use of gravitational flows, and restriction of pumping to low-cost power periods (e.g. overnight pumping) as far as possible. This work concentrates on the better utilisation of the pumps because they represent one of the largest operational costs of the network. This also means that the reduction of chlorination requirements comes second in the optimisation task list. However, minimising these requirements does not imply reducing water quality criteria. Even if pumping operations are optimised, the new sequence has to ensure that a sufficient chlorine level is provided in the treated distributed water. Chlorine is a cost-efficient, easy-touse disinfectant, effective in killing bacteria and having residual properties far better than those of ozone. Unfortunately, it produces odours that are easily recognised by customers. When solving the problem of operational optimisation of the water distribution network, efforts are made to find a compromise between sufficient chlorination (to ensure bacteriological quality) and providing the network with water which consumers find pleasant to drink.

One commonly used approach in operational optimisation problems has been the utilisation of a MINLP algorithm (e.g. Pahor and Kravanja, 1995; Grossman, 1996; Bruno et al., 1998; Zamora and Grossman, 1998; Hostrup et al., 2000). In this study, the 
necessity to achieve optimal planning within a system combining dynamic elements (volume and chlorine concentration responses) and discrete elements (pumps/valves status or routing) makes this a constrained optimisation and a challenging model predictive control (MPC) problem,

MPC techniques for constrained multivariable systems have been developed and applied by many workers (e.g. Mulholland and Narotam, 1996; Deghaye et al., 2000; and Le Lann, 1999). However, these researchers did not consider the possibility of discrete (integer) variables in their structures. MPC algorithms compute a sequence of manipulated variable adjustments to optimise future plant behaviour up to a defined time-horizon. The first input in the optimal sequence is implemented, and then the entire optimisation is repeated at the next time-step, with the horizon also moving forward one step.

The optimisation procedure used in this work is based on a model defined using a basic element, which has one input and two outputs, linked through a vessel of variable volume. All of the necessary physical properties can be defined so that the model allows the simulation of all the elements of the network: pipes, reservoirs, valves or pumps. The overall network is defined by linking several basic elements.

To achieve the two objectives, a penalty function and constraints are defined, which take into account all of the characteristics peculiar to the water distribution system being modelled. This penalty function is minimised over a defined time horizon, with input and output variables obeying the constraints.

This non-linear optimisation is realised in real-time, as a predictive control problem. It requires therefore the anticipation of a consumer demand profile, which is obtained from historical data stored by the SCADA system monitoring the network. This optimisation is realised in real-time, in the sense that the actual optimised volumes in the reservoirs and chlorine concentrations (when available) are fed back before the calculation at the next time-step takes place.

The ability of the MINLP solution to handle integer choices (e.g. pumps on or off) also allows the assessment of topological changes (e.g. extra reservoirs, pumps or link-lines). Maximum flows supported by the pipes, reservoir volumes, and pump characteristics are some of the physical measurements required to construct the model.

This project was developed as a pilot study to reveal potentials and requirements for a full simulation/optimisation application on the Durban water distribution system. Thus, the simulated case study presented here includes the variety of features that can be found in the full system. The paper describes the model used to define the network and presents the MINLP method, and then deals with the results of the case study. Finally, it ends with a description of the future work to be undertaken, along with some concluding remarks.

\section{Theory}

The choice of a mixed integer non-linear programming approach to solve this problem has been combined with the creation of a standard element. This element is used to build the different parts of the water distribution system, taking into account all of the equations and constraints determining the system.

\section{Standard element used in the MINLP optimisation}

In the course of this project, two different strategies were developed, as the results of the first were unsatisfactory.
Initially, an accurate and comprehensive representation of the physical phenomena taking place in the network was developed. This calculated flows strategy, based on friction losses and available heads, gave a detailed pressure-balanced solution which was found to be unnecessary. The details of this approach are published in Biscos et al. (2002). By observing typical operating sequences on the system, it was realised that the key issue was the sequencing of pumps located on the network trunk.

A new approach, which we have termed the available flow model, has been developed according to these observations. It employs the concept of a fixed maximum flow in a pipe section, depending on whether a pump is active or not. The current flows are calculated as the product of the maximum flow defined for the line and the actual valve position in this particular line.

The new hydraulic model proposed here simplifies the pipe network hydraulics. All the network components are still represented, using a standard modelling element, but the flows in these elements are determined in a linear way, using a valve opening or a pump status. Essentially, the system hydraulics are solved using a mass balance approach, without taking into account pipe friction. This new approach removes all the problems of non-linearity linked to the use of pressure balances in the equations and simplifies the amount of data to be provided for the model. As a result, the definition of input and output pressures, and line and valve coefficients are no longer required.

The general modelling element created for the "available" flows strategy and used in the optimisation of the network is shown in Fig. 1. Generally speaking, it has one input and two outputs, and one optional auxiliary input used to simulate an external water supply or a chlorine re-dosing point on the network. The inputs and outputs are linked through a vessel of variable volume. This vessel has a liquid volume $V$, volume setpoint $V_{S P}$, chlorine concentration $C$, and chlorine concentration setpoint $C_{S P}$. A chlorine decay constant $(k)$ has to be defined for each element.

Figure 1 shows all the available options for a model element. However, not all options for every node of the network will be used. For instance, there may be only one outlet instead of two. The two output legs can be fitted each with a valve or a pump, defined either as continuous or binary. Each leg is consequently associated respectively with valve openings or pump status (respectively $X_{A}$ for leg $A$, and $X_{B}$ for leg $B$ ); and "available" flows (respectively $f_{\text {available } A}$ for leg $A$, and $f_{\text {available } A}$ for leg $B$ ). In this model, the "available" flows (defined earlier as the maximum flows supported by each output lines) are equivalent to the flows provided by each output leg, once the valve fitted on the leg is fully opened. If there is an online pump in the same line, its contribution is translated into an additional flow ( $\Delta f_{\text {pump } A}$ and $\left.\Delta f_{\text {pump } B}\right)$, added to the initial "available" flow. The auxiliary flow being optional, its properties, flow $\left(f_{\text {aux }}\right)$ and chlorine concentration $\left(C_{\text {aux }}\right)$ are defined separately.

The variable values associated with a node may be considered as fixed or variable. Those that are variable will be subject to the set constraints and adjusted by the optimisation algorithm.

\section{Definition of network components using standard elements}

A closer look at this model is necessary to understand how the different elements of the water supply system, whatever their type, are defined using the same standard element.

\section{Reservoir}

The definition of a reservoir is illustrated in Fig. 1. A storage reservoir is represented as an open vessel with a variable volume. 


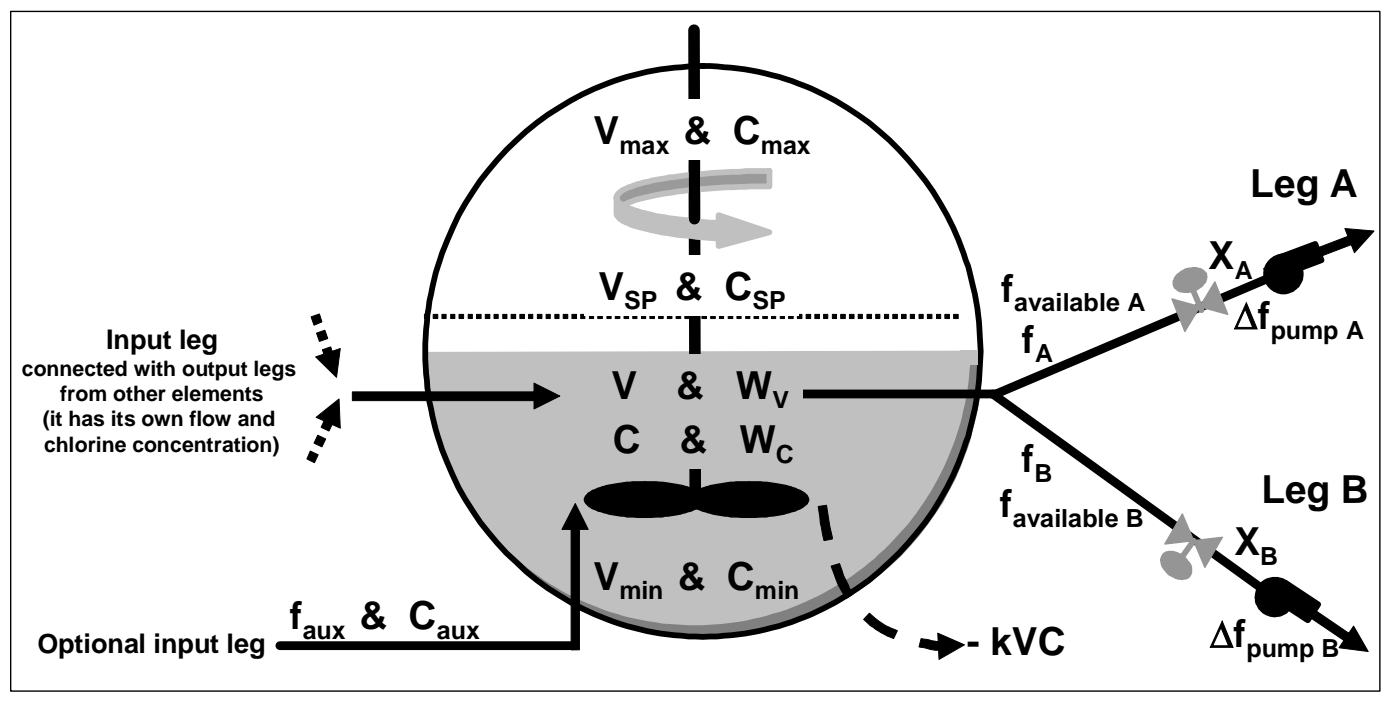

Figure 1

Standard modelling element used in MINLP optimisation of the network

\section{Pipe offtake}

To represent a pipe offtake, the volume is fixed to zero or a positive value.

\section{Valve}

The valves are intrinsic equipment on each output leg of any standard element. When parts of the network are free of valves, the user has to specify a fixed opening value corresponding to a fullyopened valve (i.e. $X_{A}$ or $X_{B}=1$ ). The model has been created so that it can take into account two types of valves: discrete or continuous. In the first configuration, the valve position variables can only access 0 or 1 values, whereas in the second case, they can access the full range between 0 and 1 . For discrete valves, the initialisation is either fully-shut or fully-open, but for continuous valves, initialisations have to correspond to a position which allows the network to reach the initial flow going through the line. These initial flows are defined separately.

\section{Pump}

The pumps are optional equipment on each output leg of any standard element, which are added by giving specific values to the valve position variables (in this particular case, $X_{A}$ and $X_{B}$ must be equal to 4 or 5). Depending of the values chosen for $X_{A}$ or $X_{B}$, the pumps are initialised in the off position (for $X_{A}, X_{B}=4$ ) or in the on position (for $X_{A}, X_{B}=5$ ) position, but the valve on the corresponding leg is always necessarily opened fully. When a pump is added, its contribution towards the flow in the line has to be defined, as well as the head of the pump. The head is taken into account in the calculation of the general electrical consumption resulting from pumping operations.

\section{Problem specification table}

Because the network consists of a combination of standard elements, an entire network problem is specified in a single table. Initialisations have to be provided for valve positions, pump status, flows, storage reservoir volumes and chlorine concentrations. Network topology, volume setpoints and constraints on reservoirs as well as chlorine concentration setpoints and available flows are fixed parameters, also defined in this table.

\section{The equations}

For every standard element in the network, several equivalence constraints must apply. Volume and chlorine balances, and constraints on physical properties are amongst the numerous equations to be defined.

\section{Flow balance}

Calculations of output flows are obtained by multiplying the current optimised valve position for the considered output leg by the available flow defined for the line. However, the flow balance equations differ slightly depending on the valve or pump configuration chosen for each output leg.

When a fixed or a binary valve is defined for one of the output legs, the flow balance equation can be written as follows:

$$
f_{A}<X_{A} \times f_{\text {available } A} \text { for leg } \mathrm{A}
$$

The available flows are fixed parameters corresponding to maximum possible flows where valves are fully open, and these are obtained in practice from reservoir volume derivatives. Thus, the calculated output flows must be lower than or equal to the product of the valve opening and the line available flow, to allow for upstream restrictions. With the demand changing continuously in the network, flows in the line are adapted accordingly and are not always necessarily equal to their maximum possible values. Similarly, in the two first configurations, where the valve positions are either fixed or accessing only binary values, the "lower than" operator has to be used to get a feasible solution.

In a configuration where a binary valve is fitted at the entrance of a storage reservoir, the flow balance equation becomes:

$$
f_{A}=X_{A} \times f_{\text {available A }} \text { for leg A }
$$

When a line is fitted with an inline pump, the flow contribution of the pump must be added to the available flow, defined with the pump offline. The flow equations are consequently transformed in the following way:

$$
f_{A}=f_{\text {available } A}+\Delta f_{\text {pump A }} \text { for leg } \mathrm{A}
$$

In Eq. (3), $\Delta f_{\text {pump A }}$ represents the flow contribution brought by the online pump in the leg.

Finally, for a fixed flow (for a reticulation flow for instance), the valve position does not have any importance and the flow equations become: 


$$
f_{A}=f_{\text {demand } A} \text { for leg } \mathrm{A}
$$

In Eq. (4), $f_{\text {demand } A}$ represents the set user demand flows from ouput leg A into the reticulation system. Note that the equations presented in this paragraph also apply for the output leg B of any standard modelling element. In this case, the index A must be replaced by the index B in Eqs. (1) to (4).

\section{Volume balance}

The volume balance on a storage reservoir is expressed by:

$$
V_{t+d t}=V_{t}+\frac{d V}{d t} d t
$$

where:

$V_{t+d t}$ corresponds to the reservoir volume for the new optimised state

$V$ is the current volume in the reservoir

$\frac{d V}{d t} d t$ represents the volume variation between the new optimised state and the current state.

This volume variation is calculated accordingly to Eq. (6):

$$
\frac{d V}{d t}=\sum_{i=1}^{N} f_{i}+f_{\text {aux }}-f_{A}-f_{B}
$$

where:

$f_{\text {aux }}, f_{A}$ and $f_{B}$ represents respectively the input auxiliary flow and output flows of the vessel

$\sum_{i=1}^{N} f_{i}$ represents the input flow coming from other parts of the network towards the reservoir input

$N$ is an index taken equal to the total number of standard

elements within the water supply system.

Equation (6) is constant between $t$ and $t+d t$. To model a closed pipe, or vessel, the volume is fixed so a zero volume is specified and $\frac{d V}{d t}=0$ is enforced, or $V_{t+d t}=V_{t}$ for the volume balance.

\section{Chlorine concentration balance}

To evaluate the chlorine concentration inside the vessel of the element, the inventories, $V C$, and the species flows, $f C$, have to be considered. By considering that the chlorine concentration in the reservoirs is not going to change greatly during a time-step (since the control objective is to keep it within limits), a good approximation of the chlorine concentration balance is given by the following equation:

$$
V_{t+d t} C_{t+d t}=V_{t} C_{t}+\frac{d V C}{d t} \times d t
$$

where:

$V_{t+d t} C_{t+d t}$ represents the chlorine inventory for the new optimised state

$V_{t} C_{t}$ corresponds to the current chlorine inventory

$\frac{d v C}{d t}$ represents the rate of variation of the chlorine inventory between the current and the new optimised state.

This concentration variation can be expressed by Eq. (8) :

$$
\frac{d V C}{d t}=f_{\text {aux }} C_{\text {aux }}+\sum_{i=1}^{N} f_{i} C_{i}-f_{A} C-f_{B} C-k V C
$$

where:

$f_{\text {aux }} C_{\text {aux }}$ is the amount of chlorine brought into the vessel by the auxiliary input flow $f_{\text {aux }}$

$\sum_{i}^{N} f_{i} C_{i}$ corresponds to the amount of chlorine present in the

water arriving from upstream parts of the network

$N$ is an index equal to the total number of elements in the system
The term $-k V C$ represents the chlorine decay inside the vessel, where $k$ is the chlorine decay constant used to simulate the first-order chlorine decay model generally chosen for this kind of system.

An approximation of Eq. (7) is then:

$$
\begin{aligned}
\left(V_{t+d t} C_{t+d t}\right)-\left(V_{t} C_{t}\right) \approx\left(f_{\text {aux }} C_{\text {aux }}\right) d t+\sum_{i=1}^{N} f_{i}\left(\frac{C_{i_{t+d+}}+C_{i_{t}}}{2}\right) d t & -\left(f_{A}+f_{B}\right)\left(\frac{C_{i_{t+d}}+C_{i_{t}}}{2}\right) d t \\
& -k\left(\frac{V_{t+d t} C_{t+d t}+V_{t} C_{t}}{2}\right) d t
\end{aligned}
$$

\section{Constraints}

Constraints are defined:

- on valve openings $\quad 0<X_{A}<1$

$$
0<X_{B}<1
$$

- on storage reservoir volumes $V_{\min }<V<V_{\max }$

- on chlorine concentrations

inside the vessels

$C_{\min }<T<C_{\max }$

\section{Penalty function to be minimised}

To achieve the two main objectives of this project, a penalty function is defined, which takes into account all the equations, constraints and characteristics peculiar to the target water distribution network. This function is minimised over a defined time horizon:

$\phi=\left(\bar{f}^{T} \overline{\Delta P}_{P}\right) \varepsilon+\left(\bar{V}-\bar{V}_{S P}\right)^{T} W_{V}\left(\bar{V}-\bar{V}_{S P}\right)+\left(\bar{C}-\bar{C}_{S P}\right)^{T} W_{C}\left(\bar{C}-\bar{C}_{S P}\right)$

The notation... indicates a multi-dimension vector quantity, for all of the elements, at the time-steps, up to the prediction horizon. The term $\left(\bar{f}^{T} \overline{\Delta P}_{P}\right) \varepsilon$ is an economic factor, which represents the cost of pumping over the simulation period. In this expression, $\varepsilon$ is the cost of unit pump power (electrical cost), while $\overline{\Delta P_{P}}$ is the normal operating pump head and $\bar{f}$ the set of flows through the pumps while they are operated.

The term $\left(\bar{V}-\bar{V}_{S P}\right)^{T} W_{V}\left(\bar{V}-\bar{V}_{S P}\right)$ corresponds to the weighted sum of squared deviations of all reservoir volumes over all time-steps, up to the control horizon. Similarly, the term $\left(\bar{C}-\bar{C}_{S P}\right)^{T} W_{C}\left(\bar{C}-\bar{C}_{S P}\right)$ penalises chlorine concentration deviations from setpoint.

In these two expressions, $W_{V}$ and $W_{C}$ are diagonal-weighting matrices used to penalise deviations of volumes and chlorine concentrations from their respective setpoints. The volume weight values have to be defined for each reservoir of the distribution systems, while chlorine concentration weight values are defined for every single standard element. The greater these values are, the tighter the control will be. However, a tight control is always achieved at the expense of computational time.

The solution provides for the following modes on any delivery leg:

$$
\begin{array}{ll}
\text { continuous valve adjustment: } & 0<\bar{X}<1 \\
\text { binary valve adjustment: } & \bar{X}=0 \text { or } 1 \\
\text { binary pump switching: } & \text { contributes } 0 \text { or } \overline{\Delta f_{\text {pump }}}
\end{array}
$$

\section{The mixed integer non-linear programming optimisation}

Mixed integer non-linear programming solves problems where both discrete and continuous choices have to be made, and where the models that support those choices are non-linear. In general, the most basic form of an MINLP problem when represented in algebraic form is described as follows: 


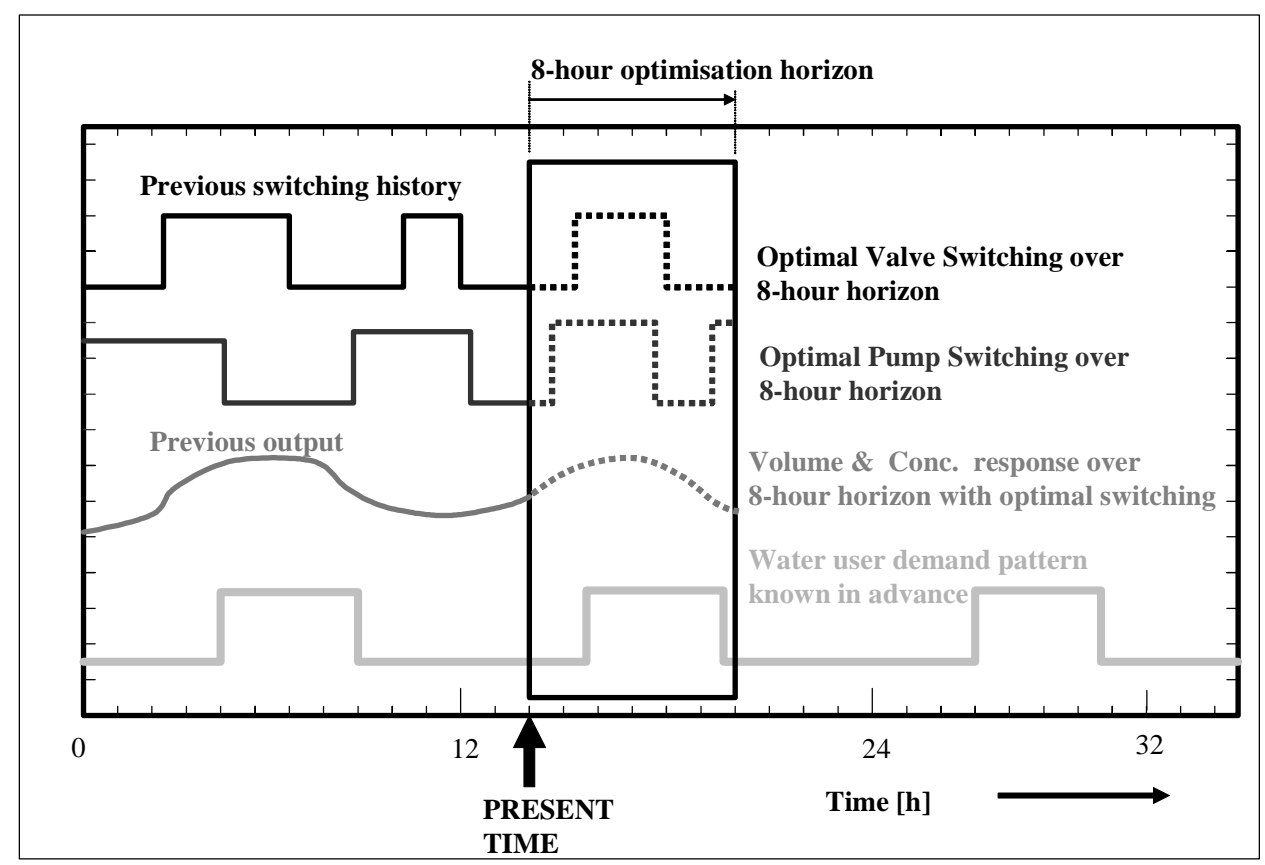

Figure 2

Predictive control over an 8-h horizon

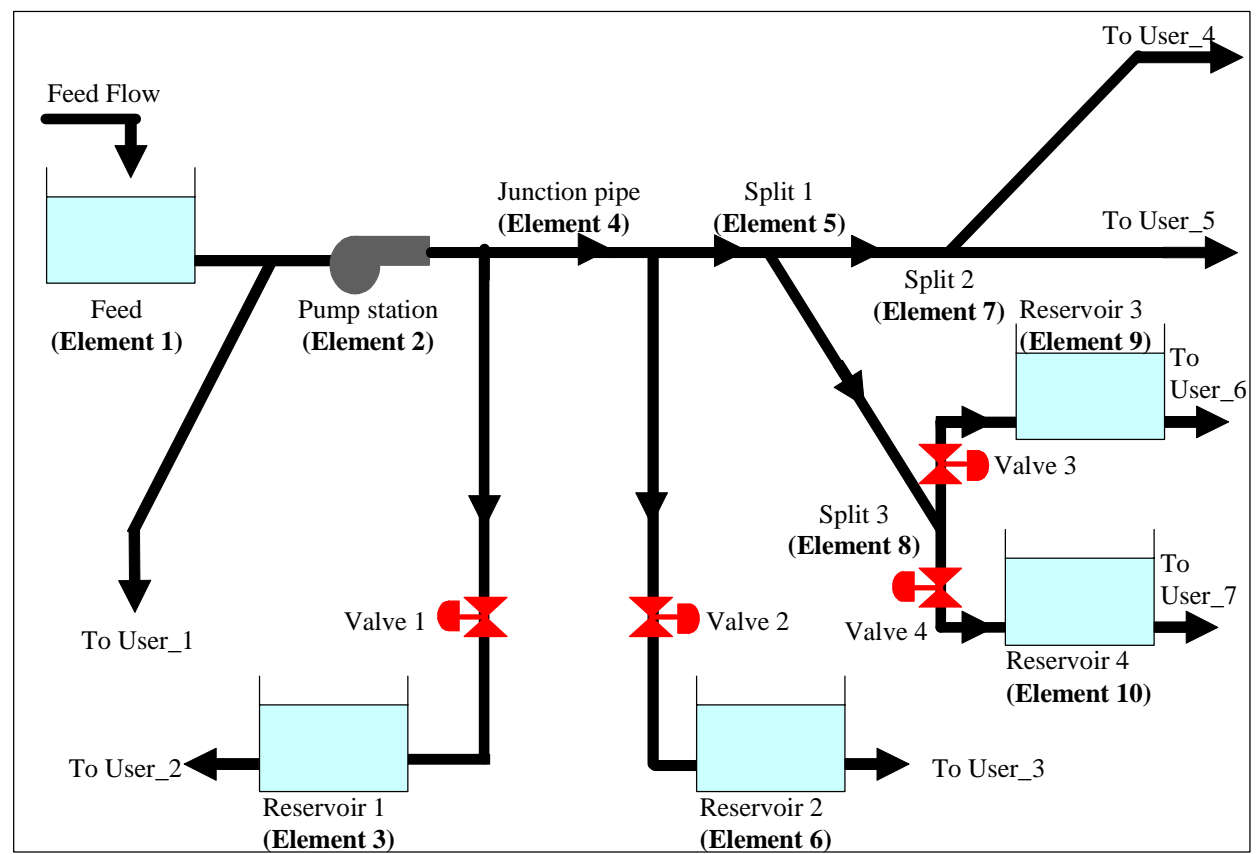

Figure 3

Case study definition
For: $\quad$ continuous variables $\mathrm{x}^{\mathrm{T}}=\left(\mathrm{x}_{1}, \ldots, \mathrm{x}_{\mathrm{n}}\right)$ and discrete variables $\mathrm{y}^{\mathrm{T}}=\left(\mathrm{y}_{1}, \ldots, \mathrm{y}_{\mathrm{n}}\right)$;

- $\mathrm{f}(\mathrm{x}, \mathrm{y})$, penalty function;

- $\mathrm{g}(\mathrm{x}, \mathrm{y})$ and $\mathrm{h}(\mathrm{x}, \mathrm{y})$, constraints;

the optimisation problem:

$\operatorname{Min} f(\mathrm{x}, \mathrm{y})$ subject to $\mathrm{g}(\mathrm{x}, \mathrm{y})=0$ and $\mathrm{h}(\mathrm{x}, \mathrm{y})>0$

is called a mixed integer non-linear programming problem (MINLP) for the general case where functions $\mathrm{f}(\mathrm{x}, \mathrm{y}), \mathrm{g}(\mathrm{x}, \mathrm{y})$ and $\mathrm{h}(\mathrm{x}, \mathrm{y})$ are nonlinear.

The continuous variables in the above could for instance describe flow rates, volumes, concentrations or design parameters of the standard elements. The discrete variables, often binary variables, may be used to describe the topology of a process network or to represent the status of pumps and valves. In general, the major empirical guideline for a good MINLP formulation is to keep the problem as linear as possible. The approach based on pressure balances was strongly non-linear (Biscos et al., 2002), so an obvious advantage was obtained in moving to the available flow model.

\section{Means used to solve the optimisation problem}

Use is made of algorithms and computer codes developed since the 1960s. A popular environment that uses these algorithms and codes 
Figure 4

Different electricity cost patterns

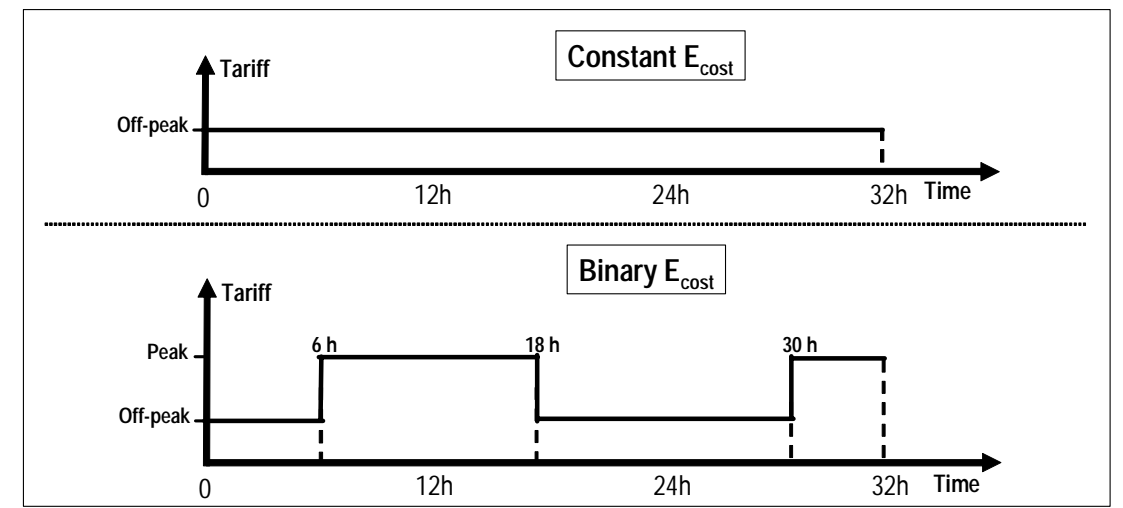

is the general algebraic modelling system (GAMS), which provides a flexible framework for formulating and solving MINLP optimisation problems (Brooke et al., 1998). It is a high-level modelling system for mathematical programming problems. It consists of a language compiler and a set of integrated high-performance solvers. GAMS is tailored for complex, large-scale modelling applications, and allows users to build large maintainable models that can be adapted quickly to new situations. The system models problems in a highly compact and natural way. The user can change the formulation quickly and easily, switching from one solver to another. In the present work, use is made of MATLAB to precompute necessary working arrays, and generate the programme which will subsequently be interpreted by GAMS to obtain the solution.

The optimisation was formulated as a predictive control problem with a moving horizon $8 \mathrm{~h}$ ahead of present time (Fig. 2). The sampling period is fixed to equal periods of $1 \mathrm{~h}$, which gives a good compromise between the accuracy of the optimisation, the amount of data to provide and the computational time. In the initial test, the solution begins at $0 \mathrm{~h}$ and proceeds to $24 \mathrm{~h}$. Thus a total of $32 \mathrm{~h}$ of known flow data (user flows from all of the supplied reservoirs) have to be provided, so that the algorithm can always look ahead at future demands over the $8 \mathrm{~h}$ horizon. These data lead to the optimal valve and pump switching which then give optimal responses for the volumes and chlorine concentrations.

\section{Case study}

A case study was created to check that the basic principles of the model give logical and reliable results. It was defined with 5 storage reservoirs, four binary valves, one pump-station, four connections to consumers and four network splits. In total, ten standard elements were combined together. The topology of this case study is presented in Fig. 3.

Attention was paid to two particular aspects: minimising of pumping costs as well as the maintenance of a target chlorine concentration in storage reservoirs and at final delivery points.

\section{Optimisation of pumping operations}

The main objective here is to reduce the pumping costs to their minimum by using gravity where possible, and favouring pump use during off-peak periods, which have a lower unit cost of electrical power. Each storage reservoir is defined with a volume setpoint, which it tries to satisfy using valve arrangements and flow redirection from upstream parts of the water supply system. Apart from this, the minimum and maximum level constraints must be complied with.
Two separate simulated experiments were conducted with different electricity cost structures. The first pattern was called "constant electricity cost" and uses the same electricity cost factor $e$ over the total length of the simulation interval. The second simulation uses a varying $e$ factor which is a combination between a low value used during off-peak periods, and a high value used during peak electricity consumption periods. The different electricity cost patterns are presented in Fig. 4. For the pattern using a binary electricity cost, the off-peak periods are defined between the beginning and the $6^{\text {th }}$ hour of the simulation, as well as between the $18^{\text {th }}$ and $30^{\text {th }}$ hour. From the $6^{\text {th }}$ to the $18^{\text {th }}$ hour and from the $30^{\text {th }}$ to the $32^{\text {nd }}$ hour, a high electricity cost is used to match the peak periods.

The chlorine concentration aspect is not studied in this particular example.

\section{Maintenance of a target chlorine concentration}

The case study was designed to highlight the ability of the optimisation package to handle chlorine concentration problems. For this simulation, an initial high chlorine concentration was defined in the feed reservoir, whereas a lower chlorine concentration was chosen for the rest of the network. By choosing a high firstorder chlorine decay constant in all the demand reservoirs, it is hoped to get quick changes in the chlorine concentration despite the short time of simulation. As the chlorine concentration in the demand reservoirs is initialised far from the setpoints, valve adjustments will have to be made to satisfy the objective.

The electricity cost influence is not studied in this particular example.

\section{Results and discussion}

Results and associated comments for both simulated experiments are presented in the following paragraphs.

\section{Optimisation of pumping operations}

Recall that in the case study, no specifications were required on chlorine concentration.

Figure 5 presents variations of the feed reservoir input and output flows. On this diagram, the plot of the pump status against time has been added. This graph clearly shows that the pumping occurs all over the simulation period, and that the pump is switched on depending on volume specifications entered for the five reservoirs. As a matter of fact, there are no limitations on the use of the pump here. Figure 6 now presents results obtained when a binary 


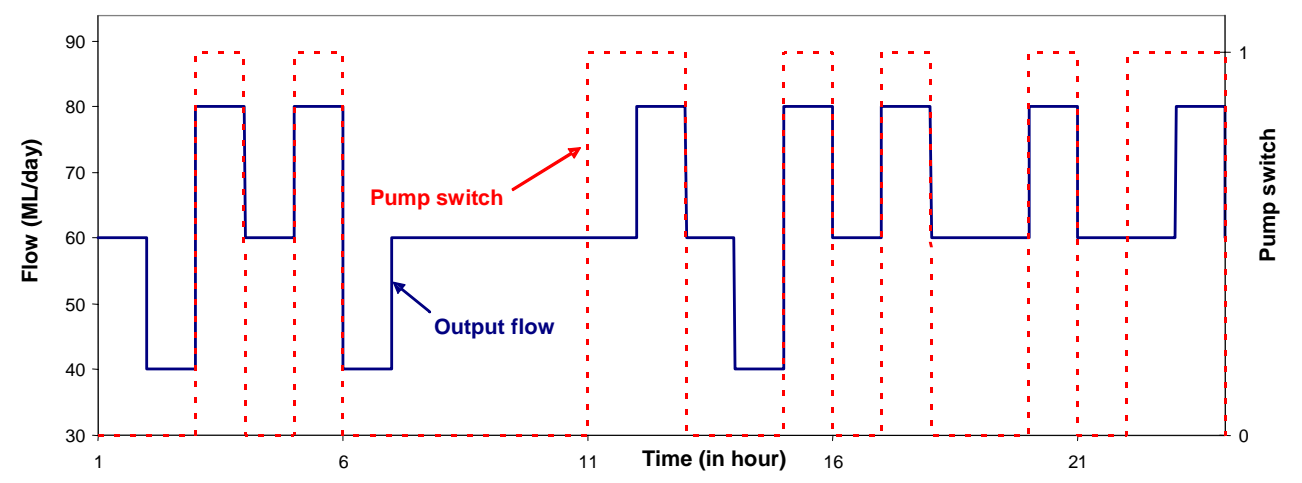

Figure 5

Feed reservoir output flow and pump status for a constant electricity cost structure

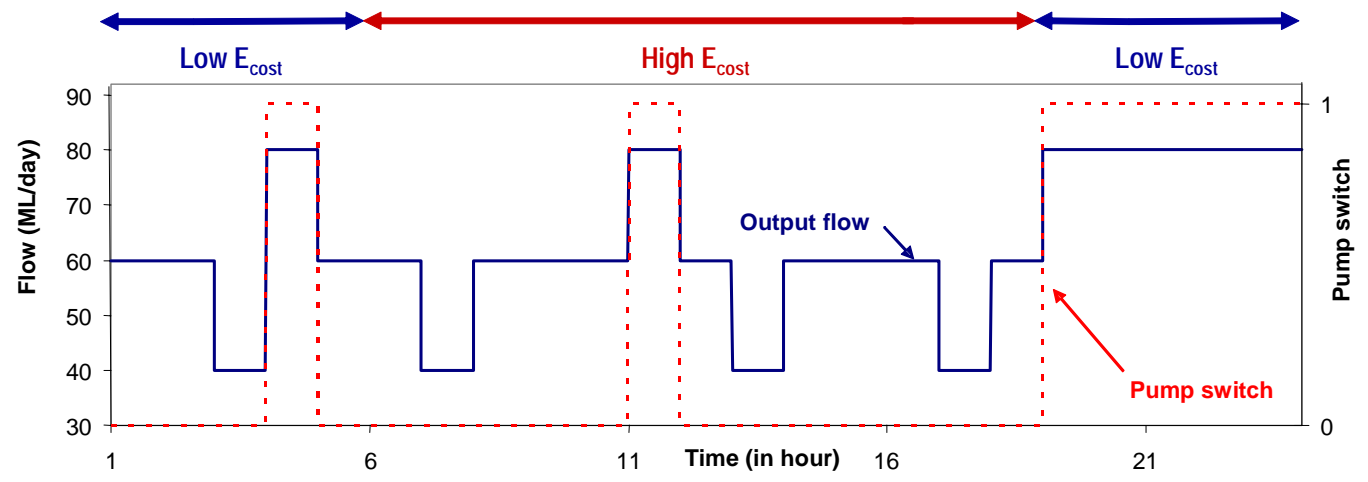

Figure 6

Feed reservoir output flow and pump status for a binary electricity cost structure

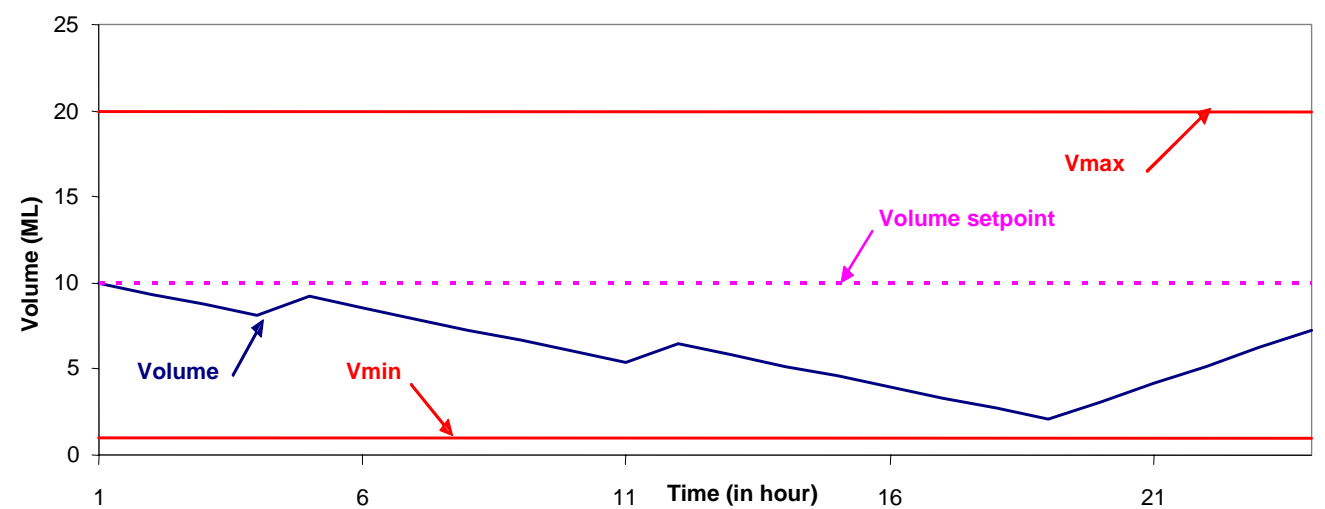

Figure 7

Storage reservoir number 2 -

Evolution of volume against time

structure is applied to the electricity cost.

Here, the pump switching appears a lot less oscillatory than with a constant electricity cost. It is clear that the pumping occurs mainly during low-cost periods; pumping during high-cost periods occurs only in the case of an emergency situation. In this particular example, it can be observed that the pump switches on during the peak period but only from the $11^{\text {th }}$ to the $12^{\text {th }}$ hour of simulation: it responds to the detection of an emergency situation in the reservoir 2 , whose volume is dropping quickly towards the minimum emergency volume, as shown in Fig. 7.

The lowest volume is only reached at the $18^{\text {th }}$ hour of the simulation. However, the pump tries to compensate for this trend from the $11^{\text {th }}$ hour, which means using high-cost power. This switching is compulsory as the volume would have reached, and probably crossed, its lowest authorised value $7 \mathrm{~h}$ later, if the change in the pump status had not occurred. It is basically an anticipation phenomenon, made possible by the use of a predictive control approach in the model. Such early action would obviously also have advantages for the setpoint tracking.
In this example, for the particular weights chosen, the penalty incurred from the electricity cost in the peak periods clearly outweighs any benefit of attempting to keep the reservoir level closer to setpoint. Since it helps to keep the volume closer to its setpoint, the use of the pump for $1 \mathrm{~h}$ in the peak period is enough to ensure that the next pump use only becomes necessary in the next off-peak period. At that time it becomes essential to pump again to avoid infringing the emergency level, though more sustained pumping is observed to bring the level closer to setpoint, and to position the system so as not to have to pump in the next high-tariff period.

It should be noted that the capacity of the main line is not large enough to ensure filling more than one storage reservoir simultaneously. Consequently, reservoir 3 and 4 input valves are shut from the $18^{\text {th }}$ to the $24^{\text {th }}$ hour to favour the supply of reservoir 2 as shown in Fig. 8.

This results in a decrease of the volumes of these two reservoirs as presented in Fig. 9. To summarise, the optimiser determines priorities when decisions are taken concerning the best time and the 
Figure 8

Storage reservoirs number 3 and 4 - Input/ outflow flows and valve adjustments
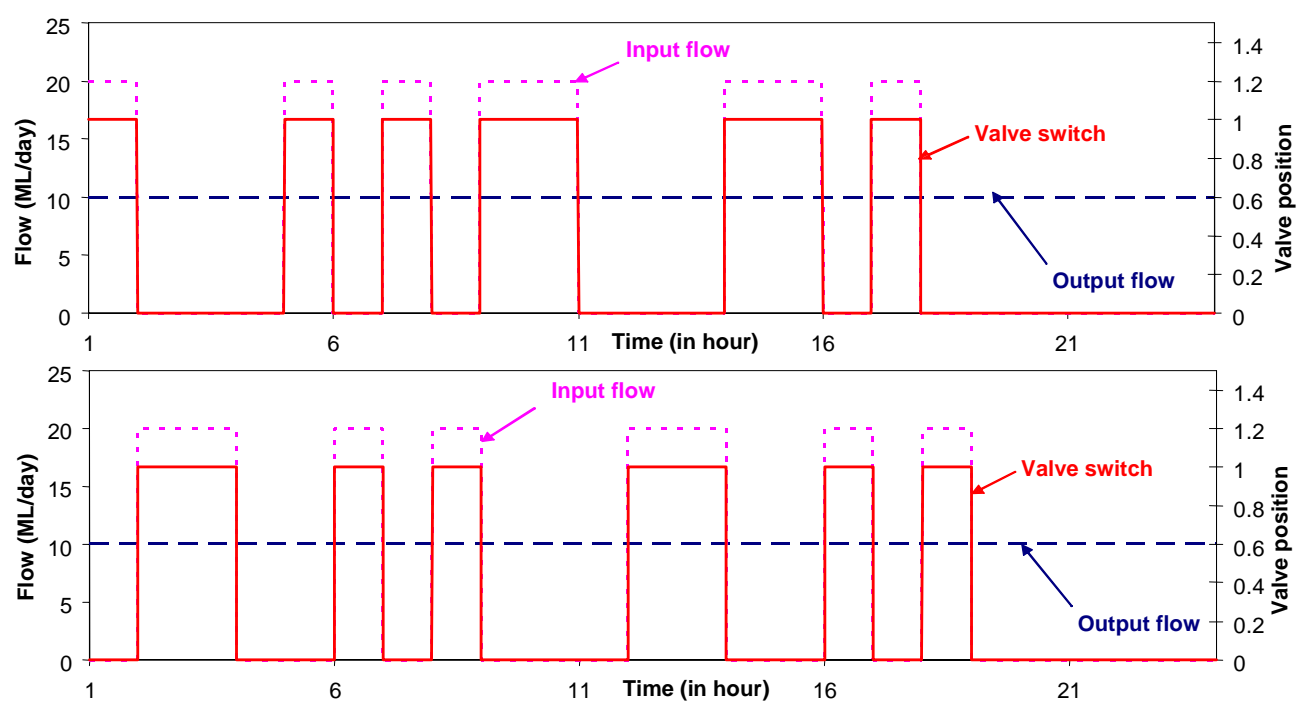

Reservoir 3:

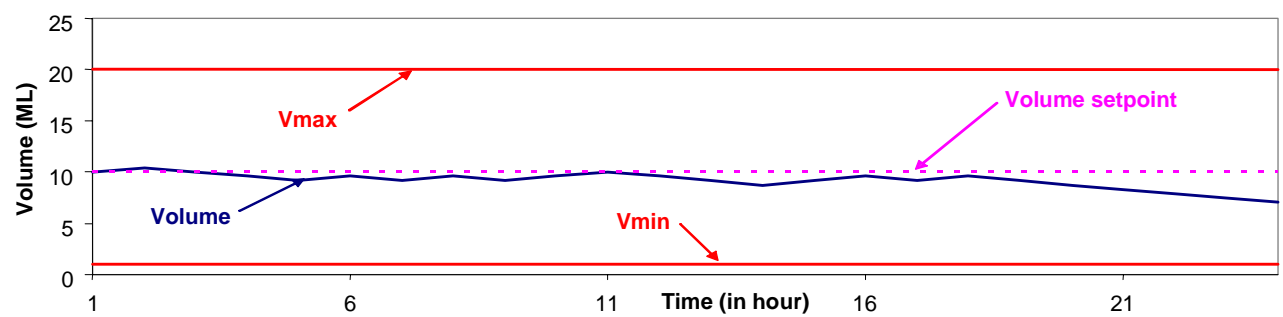

Reservoir 4:

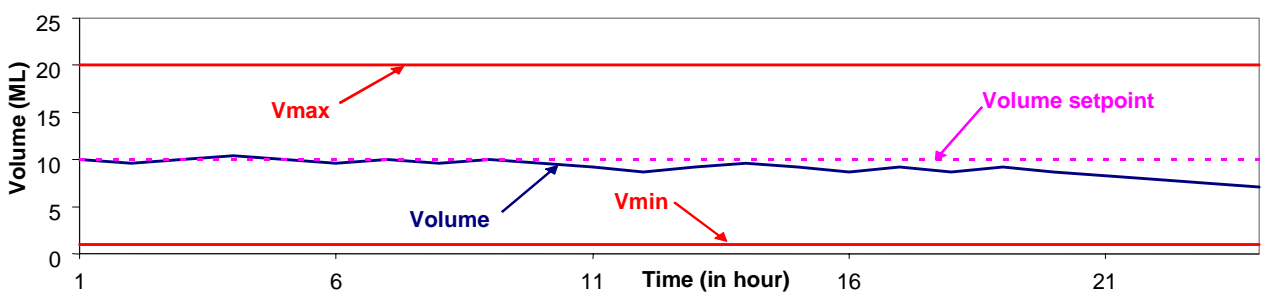

best sequence of filling of reservoirs. In this particular example, this sequence is preferred as reservoir 3 and 4 volumes are far from their emergency bounds, so their supply is stopped for a period to benefit reservoir 2 .

To conclude with this test, results occurring at these two particular storage reservoirs can also be employed to show the effect on valve constraints, with valve positions remaining in the $[0,1]$ interval, and accessing only binary values.

\section{Maintenance of a target chlorine concentration}

In this particular case study, electricity cost has no influence.

Figure 10 represents the variation of volume and chlorine concentration against the time, in reservoir 2 . One can see clearly that the chlorine objective is satisfied over the simulation period, with the chlorine concentration remaining close to the setpoint. As shown in Fig. 11, this objective is once again achieved by valve adjustments, valve positions remaining in the interval $[0,1]$, accessing only binary values. The decrease in chlorine concentration occurring during the first $10 \mathrm{~h}$ of simulation, and visible in Fig. 10, corresponds to the natural chlorine decay, which was defined with a first-order rate constant of $0.04 / \mathrm{h}$. Indeed, no water is provided in the reservoir before the elapsed time becomes equal to $10 \mathrm{~h}$ (as the input flow and valve adjustments graphs show in Fig. 11). This evolution is due to a strong demand of water in other parts of the network at the same time, and particularly on the storage reservoir number 1 . Once the valve switches on, the concentration in this reservoir immediately jumps to a level close to its setpoint, as the flow provided in the reservoir is large (close to $40 \mathrm{Ml} / \mathrm{d}$ ), and with a high chlorine concentration of $3 \mathrm{mg} / \mathrm{l}$.

After the $14^{\text {th }}$ hour, the chlorine concentration stabilises around the setpoint, but the gap between the volume in the reservoir and its setpoint is still significant. The optimiser consequently asks for new valve adjustments to have this gap reduced (in other words, providing more water to the reservoir). As the volume approaches its setpoint, it also creates increases in chlorine concentration, which now widely overshoots the setpoint. At this stage, the gap between chlorine concentration and its setpoint has more weight than the one between the volume and its setpoint. So the input valve shuts once again, meaning the chlorine concentration optimisation is favoured against the volume setpoint tracking.

To summarise, when chlorine is introduced in the simulation, accuracy is lost in the tracking of volume setpoint, because the central objectives are in conflict. Additional water inflow to raise the volume closer from setpoint would drive the chlorine concentration too high above setpoint. 


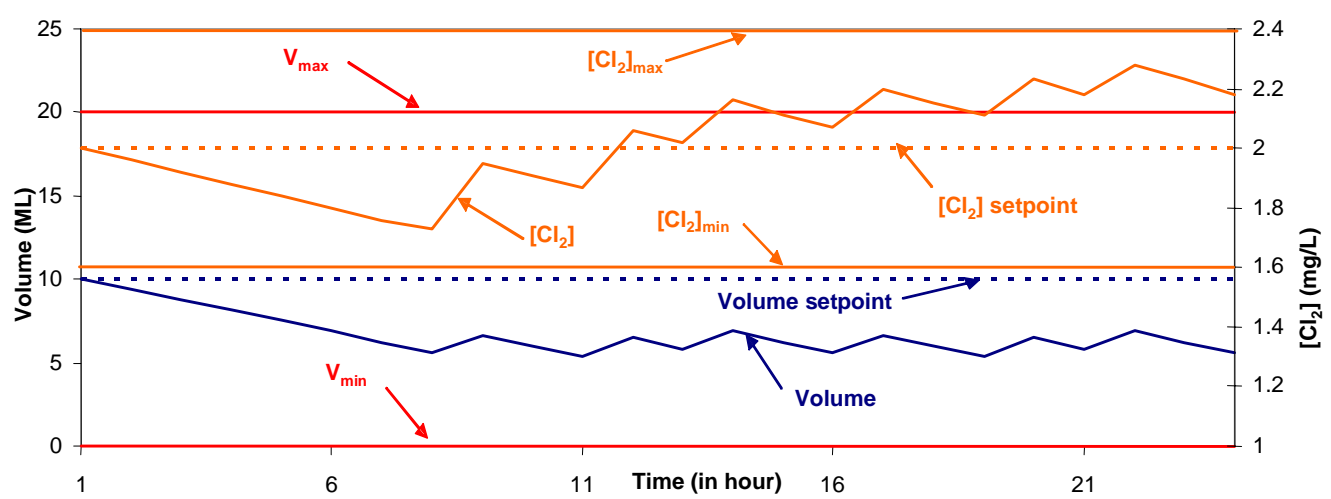

Figure 10

Storage reservoir

number 2 - Volume and

chlorine concentration

against time

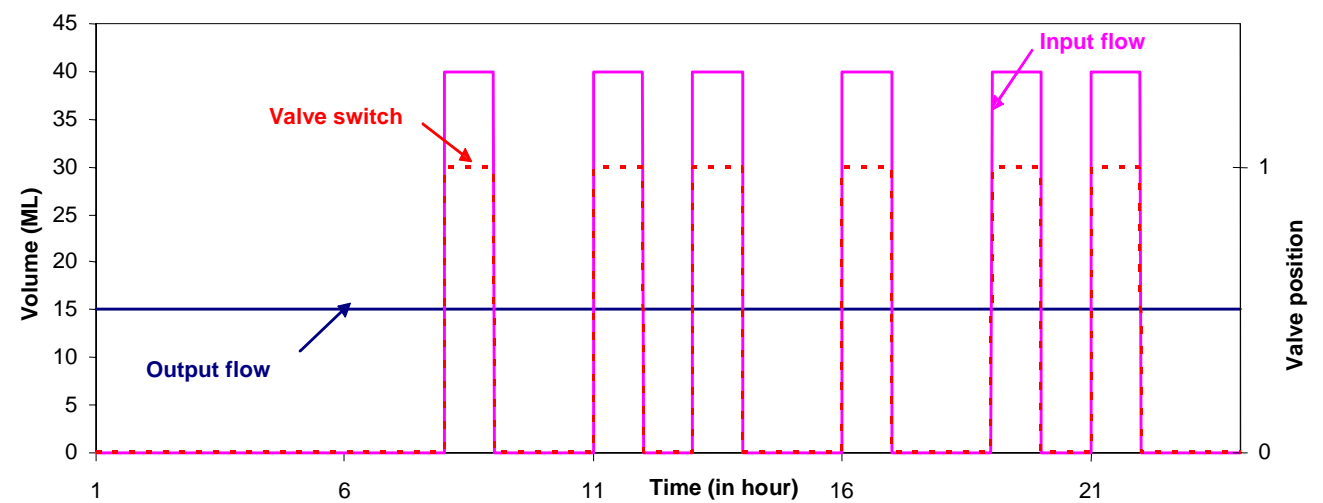

Figure 11

Storage reservoir number 2 - Input/ outflow flows and valve adjustments

\section{Future work and conclusions}

The development of the new approach, based on maximum available pipe flows rather than pressure drops, has made possible the achievement of more robust results on a 10-element case study. Since the first submission of this paper for publication, several more tests have been conducted to conclude the validation of the general methods that were defined inside the new simplified model. For instance, by providing more computational resources, it has been possible to extend the predictive control problem to a more realistic prediction window of $12 \mathrm{~h}$, with total simulation time equal to $48 \mathrm{~h}$. Similar results to those presented here have been obtained. With these tests completed, the model has been validated for application to a large-scale application, within the Durban water distribution network.

\section{Future work}

The next step of this research consists in collecting data from eThekwini Water Services, so that this simple configuration can be extended to build the real target application. It will require an accurate representation of the available flows in the lines, the acquisition of the exact network topology (storage reservoirs, valves and pumps positions), and the determination of the storage reservoir capacities. Consumer demand patterns at reticulation points are also required to determine the optimised sequence of operation. These can be easily be determined by statistical analysis of logged data, obtained by the SCADA system operated at eThekwini Water Services.

The targeted application is the Southern Aqueduct, a significant subsystem of the water distribution supply operated by eThekwini Water Services. This subsystem extends over a wide area and provides Durban's southern areas with potable water. It consists of about 20 storage reservoirs, more than five pump- stations, and of numerous valves and pipes linked together.

To apply the new "available" flows strategy on the Southern Aqueduct, and more generally on an existing infrastructure, some difficulties have to be overcome. The new approach developed in this paper, while simpler and easier, does not provide an accurate model of the hydraulic behaviour of a complex water distribution system. Such a simplified model can only be relied upon if validated using a full hydraulic model of the system. This explains why the authors recommend the use of the public domain software package ePANET (Rossman, 2000), in combination with the "available" flows approach.

ePANET is widely accepted as the world standard in hydraulic and water quality modelling of water distribution systems. Most of the existing water distribution systems have been modelled within ePANET, the Southern Aqueduct being one of these. However, ePANET cannot be used alone to meet the objectives of this project, since it is not an optimisation tool. An ePANET version of the Southern Aqueduct has been recently validated after an accurate calibration done by staff from eThekwini Water Services. In this approach, this file is used in the preliminary phase of the optimisation to determine accurately the values of the "available" flows appearing in the new version of the model presented here. However, to verify the calibration of the model further, it is also recommended to check, at the end of a simulation, that the rates at which the reservoirs fill are similar in both the model and in reality. If these rates present strong differences, it may be necessary to re-evaluate the pattern of available flows for the lines that cause the problems.

\section{Conclusion}

The two different problems treated in this case study are good examples of problems encountered within real bulk water network distribution networks. It is clear that predictive control techniques, with a moving optimisation horizon, offer an effective means of 
dealing with the problem of optimally sequencing reservoir transfers. Therefore, the employment of optimisation techniques will, at a minimum, provide a set of policies for an efficient bulk water distribution network operation, but could potentially result in a real-time automatic sequencing system.

The development of real-time automatic sequencing will depend on the results obtained in the next phase of this project (i.e. extension of the use of the new tool presented in this paper, from the case study developed here to an existing infrastructure). It is hoped that the combination of techniques used for this project would help overcome the "curse of dimensionality" linked to nonlinear programming techniques. This expression refers to the need to limit the size of the problem optimised by NLP, since the computational requirements become extensive as the size of the system grows.

To implement this model online, it may be necessary to run offline the approach presented in this paper for different plant conditions, representing the most common operating conditions of the system. The optimised operating conditions resulting from these offline optimisations would then be stored in a table for further use. As operating conditions vary, the next step would be to choose online the most similar settings amongst one of these sets. As no other estimation occurs other than determining which of the offline runs represent the most closely the actual plant conditions; the controller should adapt quickly to changing process conditions.

\section{Acknowledgements}

The authors would like to thank the Water Research Commission, the National Research Foundation (NRF) of South-Africa, the Centre National de la Recherche Scientifique (CNRS) of France, the University of Natal Research Committee and the eThekwini Metro staff.

\section{References}

ALLA P and JARRIGE PA (1988) Optimal control of the West Parisian area water supply network. In: B Coulbeck and CH Orr (eds.) Computer Application in Water Supply. Volume 2: Systems Optimization and Control. Research Studies Press Ltd. 376-391.

ALPEROVITS E and SHAMIR U (1977) Design of optimal water distribution systems. Water Resour. Res. 13 (6) 885-900.

BISCOS C, MULHOLLAND M, LE LANN MV, BROUCKAERT CJ, BAILEY R and ROUSTAN M (2002) Optimal operation of a potable water distribution network. Water Sci. and Technol. 46 (9).

BRION LM and MAYS LW (1991) Methodology for optimal operation of pumping stations in water distribution systems. ACSE J. Hydraul. Eng. 117 (11) 1-19.

BROOKE A, KENDRICK D, MEERAUS A and RAMAN R (1998) GAMS: A User's Guide. GAMS Development Corporation.

BRUNO JC, FERNANDEZ F, CASTELLS F and GROSSMAN IE (1998) A rigorous MINLP model for the optimal synthesis and operation of utility plants. Trans IChemE 76 (Part A) 246-258.

CARPENTIER P (1983) Décomposition et Aggrégation dans la Conduite Optimale d'un Grand Réseau de Distribution d'Eau. PhD Thesis, Ecole Nationale des Mines de Paris. Paris, France.

CEMBRANO G, WELLS G, QUEVEDO J, PÉREZ R, ARGELAGUET R (2000) Optimal control of a water distribution network in a supervisory control system. Control Eng. Practice 8 1177-1188.

CHASE DV and ORMSBEE LE (1989) Optimal pump operation of water distribution system with multiple storage tanks. Proc. 1990 ASCE Conf. on Water Resour. Planning and Manage. ASCE, New York 733-736.

COULBECK B (1977) Optimization and Techniques in Dynamic Control of Water Distribution Systems. PhD Thesis, Univ. of Sheffield, United Kingdom.
COULBECK B (1988) A review of methodologies for modelling and control of water supply. In: B Coulbeck and CH Orr (eds.) Computer Application in Water Supply. Volume 2: Systems Optimization and Control. Research Studies Press Ltd. 80-109.

CUNHA MC and SOUSA J (1999) Water distribution network design optimization: Simulated annealing approach. J. Water Resour. Planning and Manage. 125 (4) 215-226.

CREASEY JD (1988) Pump Scheduling in Water Supply: More Than a Mathematical Problem. In: B Coulbeck and CH Orr (eds.) Computer Application in Water Supply. Volume 2: Systems Optimization and Control. Research Studies Press Ltd. 279-289.

DEGHAYE SW, GUIAMBA I and MULHOLLAND M (2000) Enhanced dynamic matrix control applied to liquid-liquid extraction. Proc. S. Afr. Inst. of Chem. Eng., $9^{\text {th }}$ Natl. Meeting, Secunda. 9-12 October.

DUAN N, MAYS LW and LANSEY KE (1990) Optimal reliability-based design of pumping and distribution systems. J. Hydraul. Eng. 116 (2) 249-268.

EIGER G, SHAMIR U and BEN-TAL A (1994) Optimal design of water distribution networks. Water Resour. Res. 26 (4) 539-549.

FALLSIDE F and PERRY PF (1975) Hierarchical optimization of a water supply network. Proc. of the IEEE. 122 (2) 387-401.

FALLSIDE F (1988) Computer techniques for on-line control of water supply networks. In: B Coulbeck and CH Orr (eds.) Computer Application in Water Supply. Volume 2: Systems Optimization and Control. Research Studies Press Ltd. 313-329.

FUJIWARA O, JENCHAIMAHAKOON B and EDIRISINGHE NCP (1987) A modified linear programming gradient method for optimal design of looped water distribution networks. Water Resour. Res. 23 (6) 977-982.

GOLDBERG DE and KUO CH (1987) Genetic algorithms in pipeline optimization. J. Computing in Civil Eng. 1 (2) 128-141.

GOULDER IC and MORGAN DR (1985) An integrated approach to the layout and design of water distribution networks. Civil Eng. Systems 2 (2) $104-113$.

GROSSMAN IE (1996) Mixed-integer optimisation techniques for algorithmic process synthesis. Advances in Chem. Eng. 23 171-195.

HOSTRUPM, GANI R, KRAVANJAZ, SORSAK A and GROSSMAN IE (2000) Integration of thermodynamic insights and MINLP optimization for the synthesis, design and analysis of process flowsheets. Computers \& Chem. Eng. 25 73-83.

JOWITT PW, GARETT R, COOK S and GERMANOPOULOS G (1988) Real-Time Forecasting and Control for Water Distribution. In: B Coulbeck and CH Orr (eds.) Computer Application in Water Supply. Volume 2: Systems Optimization and Control. Research Studies Press Ltd. 329-355.

JOWITT PW and GERMANOPOULOS G (1992) Optimal pump scheduling in water-supply. ASCE J. Water Resour. Planning and Manage. 118 (4) 406-422.

KESSLER A and SHAMIR U (1989) Decomposition technique for optimal design of water supply networks. Water Resour. Res. 25 (7) 1469-1480.

KESSLER A and SHAMIR U (1991) Analysis of the linear programming gradient method for optimal design of water supply networks. Eng. Optimization. 17 (1) 1-19.

KIM JH and MAYS LW (1994) Optimal rehabilitation model for water distribution systems. J. Water Resour. Planning and Manage. 120 (5) 982-1000.

LANSEY KE and MAYS LW (1989) Optimization model for water distribution system design. J. Hydraul. Eng. 115 (10) 1401-1418.

LANSEY KE and ZHONG Q (1990) A methodology for optimal control of pump stations. Proc. 1990 ASCE Conf. on Water Resour., Planning and Manage. ASCE, New-York.

LE LANN MV (1999) Application de la Commande Avancée en Génie des Procédés, Proc. Conférence inaugurale. Colloque: Applications de la Commande Avancée, Paris. Mars 5-12.

MOUSAVI H and RAMAMURTHY AS (1999) Optimal design of multireservoir systems for water supply. Adv. in Water Resour. 23 (2000) 613- 624.

MULHOLLAND M and NAROTAM NK (1996) Constrained Predictive Control of a Counter-Current Extractor. In: Dolezal and J Fidler (eds.) System Modelling and Optimisation, Chapman \& Hall. 251-258. 
PAHOR B and KRAVANJA Z (1995) Simultaneous solution and MINLP synthesis of DAE/process problems: PFR networks in overall processes. Computers \& Chem. Eng. 19 (Suppl.) 181-188.

QUINDRY GE, BRILL ED and LIEBMAN JC (1981) Optimization of looped distribution systems. J. Environ. Eng. 107 (EE4) 665-679.

RAO HS and BREE (Jr.) DW (1977) Extended period simulation of water systems - Part A. ASCE J. Hydraul. Div. 103 (2) 97-108.

ROSSMAN LA (2000) EPANET 2 Users Manual. US Environmental Protection, Agency, Cincinnati.

SCHAAKE J and LAI D (1969) Linear Programming Dynamic Programming Applications to Water Distribution Network Design. Dept. of Civil Eng., Massachusetts Inst. of Technol., Cambridge, Massachusetts. Report no 116.

SOLANAS JL (1974) Mando en Tiempo Real De Un Sistema de Suministro de Agua. Ph.D. Thesis, Univ. Politécnica de Cataluña, Barcelona, Spain.

SOLANAS JL and MONTOLIU JM (1988) The optimal operation of water systems. In: B Coulbeck and CH Orr (eds.) Computer Application in
Water Supply. Volume 2: Systems Optimization and Control. Research Studies Press Ltd. 357-375.

TEEGAVARAPU RSV and SIMONOVIC SP (2002) Optimal Operation of Water Resource Systems: Tradeoffs Between Modelling and Practical Solutions - Research Report R3T 2N2. Dept of Civil and Geol. Eng., Univ. of Manitoba, Winnipeg, Canada.

WOOD DJ and RAYES AG (1981) Reliability of algorithms for pipe network analysis. ASCE J. Hydraul. Div. 107 (10) 1145-1161.

WOODBURN J, LANSEY KE and MAYS LW (1987) Model for the optimal rehabilitation and replacement of water distribution system components. Proc. Natl. Conf. on Hydraul. Eng. Williamsburg, Virginia, USA.

YU G, POWELL RS and STERLING MJH (1994) Optimized pump scheduling in water distribution systems. J. Optimization Theory and Applications 83 (3) 463-488.

ZAMORA JM and GROSSMAN IE (1998) A global MINLP optimisation algorithm for the synthesis of heat exchanger networks with no stream splits. Computers \& Chem. Eng. 22 367-384. 